hep-ph/9608197, SCIPP 96/31, RU - 96 /95

\title{
The Cosmology of String Theoretic Axions
}

\author{
Tom Banks ${ }^{a}$ and Michael Dine ${ }^{b}$ \\ ${ }^{a}$ Department of Physics, Rutgers University, Piscataway, NJ 08540 \\ ${ }^{b}$ Santa Cruz Institute for Particle Physics, University of California, Santa Cruz, CA 95064
}

String theory posesses numerous axion candidates. The recent realization that the compactification radius in string theory might be large means that these states can solve the strong CP problem. This still leaves the question of the cosmological bound on the axion mass. Here we explore two schemes for accommodating such light axions in cosmology. In the first, we note that in string theory the universe is likely to be dominated early on by the coherent oscillations of some moduli. The usual moduli problem assumes that these fields have masses comparable to the gravitino. We argue that string moduli are likely to be substantially more massive, eliminating this problem. In such cosmologies the axion bound is significantly weakened. Plausible mechanisms for generating the baryon number density are described. In the second, we point out that in string theory, the axion potential might be much larger at early times than at present. In string theory, if $\mathrm{CP}$ violation is described by a small parameter, the axion may sit sufficiently close to its true minimum at early times to invalidate the bounds.

$7 / 96$ 


\section{Introduction}

The invisible axion is an elegant solution to the strong CP problem. At first glance, string theory is replete with axion candidates. At weak coupling, these include the "modelindependent axion" [1] and axions which arise from internal components of $B_{\mu \nu}$ [2]. There are two problems with these axions. First, in the weakly coupled region there are no good arguments that QCD is the dominant contribution to the potential of any axion. Consider, first, the usual "model-independent" axion. While this axion respects a Peccei-Quinn (PQ) symmetry in perturbation theory, this symmetry is likely to be broken by both stringy nonperturbative effects and hidden sector dynamics. In ref. [3], it was shown that discrete symmetries could adequately suppress hidden sector contributions. However, we know of no argument that there cannot be inherently stringy PQ symmetry-violating effects of order $e^{-c / g}$. Unless $c$ was suprisingly large, these effects would be far larger than those associated with QCD. Similarly, at large radius, there are potential axions associated with the internal components of the antisymmetric tensor. These symmetries, however, are broken by world-sheet instantons, the breaking being of order $e^{-R^{2}}$. In the weak coupling picture, however, $R$ is necessarily of order 1 .

The second problem with these axions is that their decay constants appear to be incompatible with cosmological bounds [4] [5] [6]. We have recently shown that in certain strongly coupled string vacua, the first difficulty is removed[7]. The purpose of the present paper is to demonstrate that the cosmological argument is not a barrier to stringy axion models. In fact, we will present two different scenarios for cosmology and particle physics in which axions with decay constants much larger than the conventional bounds are allowed. The first scenario depends on a number of relatively obscure facts about string theory. It is conventional to estimate the order of magnitude of the decay constant of stringy axions, as well as analogous parameters for other moduli, to be the Planck scale. In fact, in a calculation done almost ten years ago, Kim and Choi [11] showed that the model independent axion decay constant in weakly coupled heterotic string theory is really $\frac{M_{p}}{16 \pi^{2}}$

1 There have been previous proposals to solve this problem. Late decaying particles, somewhat similar to the moduli under discussion here, have been considered in ref. [6] and in [8] and [9] . Weak anthropic ideas have been considered in [10]. 
or about $10^{16} \mathrm{GeV}$. So it is not unreasonable to expect large numerical factors in the relation between decay constants and the Planck mass. In the strong coupling regime, we have suggested an effect which might further lower the decay constant.

It does not seem plausible, however, that such arguments can lower the axion decay constant by the seven orders of magnitude necessary to satisfy the conventional cosmological bound of $10^{12} \mathrm{GeV}$. However, it is likely that string cosmology is not entirely conventional. Indeed, in all vacuum states explored to date, there are moduli whose masses are determined by supersymmetry (SUSY) breaking. It is usually assumed that the characteristic scales of variation of these moduli - what we might loosely refer to as their "decay constants", are of order the Planck mass. In that case, they dominate the energy density of the universe until its energy density is too low for conventional nucleosynthesis to proceed. However, as we will see, a mild retuning of the modular decay constants, similar to, but less dramatic than, that of Kim and Choi for the axion, is sufficient to solve the moduli problem. Typically this leads to a cosmology which is matter dominated from a short time after inflation until just before nucleosynthesis.

In such a context, we will see that the axion problem is greatly ameliorated. We will make the standard assumption that after inflation the axion is left stranded at a random place on its potential. We then show that the axion begins to oscillate at a time when its energy density is smaller than the total density of the universe by a factor of $\frac{f_{a}^{2}}{m_{p}^{2}}$. If the cosmic energy density is dominated by other coherent scalars (or nonrelativistic matter), this ratio remains constant until those scalars decay into relativistic matter. There is a wide range of reheat temperatures, above the nucleosynthesis temperature, for which axions with decay constants much larger than the conventional bounds do not dominate the universe before the conventional era of matter domination. Furthermore, the scenario is compatible with the idea that the coherent scalars whose decay gives rise to the Hot Big Bang are moduli fields, that the axions are the dark matter, and that gravitinos and topological objects such as domain walls are sufficiently diluted.

The question that remains is the origin of baryogenesis. We argue that the decay of the moduli may well be the source of the baryon asymmetry. Such a scenario requires dimension four baryon number violating operators. It is not consistent with the existence 
of a stable superpartner, which might be the dark matter. On the other hand, for a range of decay constants axions can be the dark matter and a stable sparticle is unnecessary. We also study a scenario in which baryogenesis arises from coherent condensates of standard model sparticle fields [12] [13]. We find that this possibility is viable if the associated directions are lifted only by very high dimension operators - or not at all.

Our second strategy for relaxing the bound on the axion decay constant depends on an unorthodox assumption about the origin of CP violation. The standard model of $\mathrm{CP}$ violation through Cabibbo Kobayashi Maskawa (CKM) phases requires a fundamental $\mathrm{CP}$ violating phase of order one. The miniscule size of $\mathrm{CP}$ violating effects in current experiments is attributed to the necessity of mixing all three quark generations, and to the small size of the CP conserving mixing angles. In such a scheme one expects generic $\mathrm{CP}$ violating phenomena to be unsuppressed. In the context of supersymmetry, one has difficulty understanding the smallness of CP-violating phases in soft breaking parameters required by phenomenology, and the smallness of $\theta$ is a mystery. There exist alternate approaches in which the fundamental CP violating phases are all small, so that the breaking of CP is everywhere controlled by a small parameter. We will review such a proposal, due to Nir and Ratazzi [14]. In this class of models, gaugino and other phases are automatically small enough, a CKM phase of conventional size explains all current data on CP violation, but the smallness of $\theta$ requires further explanation.

String theory is a theory where $C P$ is a good symmetry - in fact a gauge symmetry - which must be spontaneously broken[15]. It contains a host of CP odd fields which might provide the small breaking of [14]. Moreover it contains numerous axion candidates which can relax $\theta$ to zero. The usual objections to such a picture would be the large value of the axion decay constants, and domain walls due to spontaneous CP violation. Since CP is a gauge symmetry, the domain walls are not absolutely stable, but they may have lifetimes much longer than the age of the universe. In the conclusions we will argue that this and all other domain wall problems can be solved in models where the energy density is matter dominated until quite low scales. In such models, spontaneous symmetry breaking is frozen in at the end of inflation and the temperature never gets high enough for symmetry restoring phase transitions. 
Moreover, in a world with everywhere small, spontaneous $\mathrm{CP}$ violation, the cosmological axion problem may be significantly ameliorated. During inflation, certain inflaton fields remain displaced from their minima. As a consequence, the effective potential for all other scalars may take values significantly different than their values in the vacuum. These, passive, scalars are rapidly driven to the minima of the inflationary potential. In general, the inflationary minimum will be nowhere near the true minimum of the vacuum potential. (This is, in some sense, the origin of the "moduli problem.") Among these passive fields are the candidate axions. Their potentials can be large either if the QCD scale is large due to a displaced dilaton 16,2 or if world sheet instanton effects (in weakly coupled string language) are enhanced due to diplaced Kahler moduli. However, if the inflaton fields are all CP invariant, and CP is broken only by small effects, then the minimum of the inflationary potential for CP-odd fields like the axion will be close to its true minimum. Thus, during inflation, the axion is driven very close to its true minimum by a very large potential. It is easy to see that the postinflationary axion energy density will be of order $\delta^{2} \Lambda_{Q C D}^{4}$, where $\delta$ is the small parameter which controls CP violation. We will see that for values of $\delta$ compatible with experiment, this is small enough to significantly enlarge the allowed range of values for the axion decay constant.

In the next two sections of this paper we present the details of the two scenarios outlined above. A third section is devoted to a brief discussion of models based on eleven dimensional supergravity which sparked our reexamination of the axion bound. The final section is devoted to our conclusions.

2 The proposal of [16] suffers from a number of difficulties. These have been discussed recently in [17]. Our proposal is quite different in that, first, we explain why the early minimum coincides with the minimum at late times, and the QCD scale is assumed much larger, so that there is little or no suppression of the axion mass.

3 We will neglect the possibility that the inflationary minimum is at $a=\pi$ while the true minimum is $a=0$. It is clearly possible to construct models in which this alternative is realized. 


\section{Axions in Moduli Dominated Cosmologies}

\subsection{Moduli Ameliorate the Axion Problem}

In our first scenario, we imagine that the postinflationary universe is dominated by one or more moduli fields, with reheat temperature $T_{R}$. From the end of inflation, until energy densities of order $T_{R}^{4}$ the universe is matter dominated. The axion begins this era at a position determined by its inflationary potential, generically a distance of order one (we define the axion to be dimensionless and to have period $2 \pi$ ) from its true minimum. Initially, it contributes a negligible amount, of order $\Lambda_{Q C D}^{4}$ to the cosmic energy density. The axion remains more or less stationary until the Hubble parameter $H$ is equal to the axion mass. After this time, it behaves like nonrelativistic matter. The crossover occurs when

$$
\frac{\sqrt{\rho}}{m_{p}}=\frac{\Lambda_{Q C D}^{2}}{f_{a}}
$$

Notice that it is the reduced Planck mass $m_{p}=2 \times 10^{18} \mathrm{GeV}$ which enters this equation.

At this time, the ratio of axion to modular energy densities is of order

$$
\frac{\rho_{a}}{\rho} \approx \frac{\Lambda_{Q C D}^{4}}{\rho} \sim \frac{f_{a}^{2}}{m_{p}^{2}}
$$

The ratio retains this value until reheat, at which time the axion energy density begins to grow relative to the energy of the massless particle gas produced by the decay of the modulus. The growth is linear in the inverse temperature.

Classical cosmology begins with the era of nucleosynthesis, where the photon temperature is of order $1 \mathrm{MeV}$. In order to preserve the physics of this era, we must have $T_{R}>1 \mathrm{MeV}$. We must also arrange the correct baryon to entropy ratio, of which more below. Finally, we must ensure that the universe remains radiation dominated until the conventional beginning of the matter dominated era at $T \sim 10 \mathrm{eV}$. Since the axion to radiation ratio increases monotonically during this era, the only requirement is that the ratio must be less than or equal to one when the radiation temperature is $10 \mathrm{eV}$. Thus

$$
\frac{f_{a}^{2}}{m_{p}^{2}} \frac{T_{R}}{10 e V} \leq 1
$$

\footnotetext{
4 Actually, one needs a somewhat larger reheat temperature, of order $6 \mathrm{MeV}[18]$.
} 
Equality corresponds to the interesting case in which axions are the dark matter in the universe.

The borderline case $T_{R} \sim 1 \mathrm{MeV}$ gives an axion decay constant bounded by $6 \times 10^{15}$ GeV. Given the results of Kim and Choi [11], this seems to be right in the interesting range for superstring axions. The bound decreases like $T_{R}^{-\frac{1}{2}}$ as $T_{R}$ increases. In particular, if we take $T_{R} \geq 100 \mathrm{GeV}$, as would be required for baryogenesis at the electroweak phase transition, then $f_{a} \leq 10^{13} \mathrm{GeV}$. While this is still higher than the conventional bound, it seems a rather low number to expect to come out of string theory. Perhaps in the strong coupling vacuum described in[19][7], if the QCD axion is a boundary modulus we could obtain a decay constant this small. Dimensional analysis suggests $10^{16} \mathrm{GeV}$ for the decay constant of such a boundary axion, and numerical factors such as those of [11] could bring us the rest of the way. We have had to choose parameters at their extreme ranges of plausibility to make this scenario work. Another mechanism of low energy baryogenesis seems to be indicated.

Before enquiring what that mechanism could be, let us indicate the expectations for $T_{R}$. The modulus whose decay initiates the Hot Big Bang 5 has a potential of the form $M^{4} \mathcal{V}\left(\frac{\phi}{f}\right)$ where $\mathcal{V}$ is a bounded function. Here $M$ is "the fundamental scale of string theory". Recent developments in string duality have shown us that we know much less about the value of $M$ than we previously thought. In weakly coupled heterotic string theory, the best fit to the real world has $M$ of order the reduced Planck mass. In the strong coupling regime[7], $M$ is closer to the unification scale. Furthermore, the function $\mathcal{V}$ can vary quite a bit over moduli space. What is relevant to our present discussion is the value of $\mathcal{V}$ near the minimum of the lightest moduli (those with the lowest reheat temperature). Let us factor this value out and include it in $M$. Then, in the regime of interest, $\mathcal{V}$ is of order one or less. It is plausible that for the lightest unstable moduli the effective value of $M$ is the fundamental SUSY breaking scale : $M \sim \sqrt{F} \sim 10^{11} \mathrm{GeV}$ (corresponding to $m_{3 / 2}$ of order a few hundred $\mathrm{GeV}$ ). This appears to be the case in all

5 There may be several fields which deserve this designation. We will pretend that there is only one for linguistic convenience, but nothing that we say depends on this assumption. 
explicit models of which we are aware. Then the mass of the modulus is of order

$$
m^{2} \approx \frac{M^{4}}{f^{2}}
$$

As a model for the decay of the moduli, consider the usual dilaton, $D$. This field (canonically normalized) couples to photons through a term

$$
\mathcal{L}_{D \gamma} \approx \frac{1}{m_{p}} D F_{\mu \nu}^{2} .
$$

So the decay width is of order

$$
\Gamma \approx \frac{m^{3}}{m_{p}^{2}} \approx \frac{M^{6}}{f^{3} m_{p}^{2}}
$$

which gives a reheat temperature

$$
T_{R} \approx \sqrt{\Gamma m_{p}} \approx \frac{M^{3}}{m_{p}^{1 / 2} f^{3 / 2}}
$$

This is of order the electroweak transition temperature for $f \sim 10^{14} \mathrm{GeV}$. So, even given the results of [11, an electroweak reheat temperature seems difficult to achieve. On the other hand, if $f \sim 10^{16} \mathrm{GeV}$, the reheat temperature is of order $1 \mathrm{GeV}$, well above the nucleosynthesis bound.

It may seem troubling to contemplate such small values of $f$. These correspond to moduli masses of order 10-100 TeV, several orders of magnitude larger than the gravitino mass. One might expect that this requires fine tuning. But, as we have argued elsewhere, such large masses are almost inevitable[20]. In particular, for small values of the gauge coupling, the superpotential typically behaves as

$$
W \approx e^{-16 \pi^{2} S / N}
$$

where $S$ is the dilaton supermultiplet and $N$ is of order 4 or 5 . We have normalized the dilaton multiplet here as in [21], so that the dilaton has a canonical kinetic term at weak coupling. With this normalization, $16 \pi^{2} S$ is periodic with period $2 \pi$. The resulting potential has no minimum at weak coupling, and one must assume that there are large corrections to the Kahler potential in order to give a stable vacuum. The second derivative of the potential is then, indeed, quite large. Moreover, the large corrections to the Kahler 
potential introduce significant uncertainties in the decay rates. We have allowed for these uncertainties in our estimates above by neglecting factors of $4 \pi$, etc., which are usually included in weak-coupling based analyses, so our formulas are somewhat more optimistic than others which appear in the literature 22].

In view of these remarks, it may be correct to conclude that the cosmological moduli problem [23] is a red herring, resulting from overreliance on naive dimensional analysis. While moduli will certainly modify cosmic history above the electroweak phase transition, and quite possibly between this scale and nucleosynthesis, there is no longer a strong reason to believe that they interfere with classical cosmology.

\subsection{Baryogenesis}

However, moduli will certainly modify baryogenesis, the gravitino problem, and the axion bound. Indeed, it seems difficult to push the reheat temperature of moduli whose masses come from SUSY breaking above the electroweak scale. Thus, even if electroweak baryogenesis remains a viable option in the presence of moduli, its details will probably be modified by modular decay. This appears to be a complicated problem, and we will not explore it. Rather, following our conclusions about axions, we will now explore other possibilities for baryogenesis.

The most straightforward scenario is to assume that modular decay itself is responsible for the baryon asymmetry. This sort of mechanism for baryogenesis apparently originates with the work of [24]. Many of the important issues are reviewed in [25] The couplings by which the modulus decays may contain CP violation and baryon number violation of relative order one $\mathrm{G}$. In order to produce a baryon asymmetry, we must have another sort of baryon violating operator in the lagrangian [26]. We will see that the coefficient of this operator cannot be too small, so it is natural to assume that it is one of the allowed renormalizable baryon number violating operators in the supersymmetric standard model. It is well known that the presence of such operators is compatible with the stability of the proton and the experimental absence of neutron-antineutron oscillations [27]. It is not

6 Here we do not make the assumption of small CP violation which will dominate our discussion in the next section. 
compatible with a model of the dark matter as a stable supersymmetric particle. However, in the present context, axions can play the role of dark matter, and there is no need for a stable superpartner.

The estimate of the baryon asymmetry produced by modular decay is simple. Assume the amount of baryon number produced per decay is $A$. $A$ is the product of a loop factor (presumably of order $\frac{\alpha_{s}}{\pi}$ times $C P$-violating phases, which, given our assumptions, are of order 1). The number of massless particles produced per decay is $\frac{m_{M}}{T_{R}}$ where $m_{M}$ is the mass of the modulus. Plugging in the expected form of the modular mass and reheat temperature, we find a baryon to entropy ratio of

$$
\frac{n_{B}}{n_{\gamma}} \sim A\left(\frac{m_{p}}{f}\right)^{\frac{1}{2}} \frac{10^{11} \mathrm{GeV}}{f}
$$

For an $f$ of order $m_{p}$ this gives a result in the desired range if $A \sim 10^{-2}-10^{-3}$.

For $f \sim 10^{16} \mathrm{GeV}$, the baryon to entropy ratio is of order $A 10^{-4}$ so this mechanism seems to produce too many baryons. Of course, this is the regime in which we might expect some effect of electroweak baryon number violation, and the situation becomes much more complicated. It seems clear that the simplest model has $f \sim m_{p}$, a reheat temperature just above nucleosynthesis, baryogenesis from modular decay, and axions with decay constant $10^{16} \mathrm{GeV}$ as dark matter.

We next explore the mechanism of coherent baryon number production of ref. [12]. One can contemplate two possibilities here. The first is that among the moduli are the inflatons. In that case, we can take over directly the estimates of the baryon asymmetry from ref. [13]. In that paper, a formula was given for the baryon number per inflaton. Schematically, this can be written:

$$
\frac{\rho_{\phi}}{\rho_{I}} \approx\left(\frac{m_{3 / 2}}{m_{p}}\right)^{2 /(n-2)}
$$

where the term in the superpotential which lifts the flat direction is of the form $\phi^{n}$, and we have assumed that $m_{p}$ is the only scale. To obtain the baryon to photon ratio after reheating, one needs to multiply this result by $T_{R} / M_{I}$, where $T_{R}$ is the reheating temperature and $M_{I}$ is the mass of the inflaton. Assuming an inflaton mass of order the weak scale and a reheat temperature of order a few $\mathrm{MeV}$, this factor is about $10^{-5}$. So 
one sees that the flat direction must be extremely flat; $n$ must be at least 8 . As explained in ref. [13], one can obtain directions this flat - or even exactly flat - by means of discrete symmetries.

Alternatively, it might be that the inflaton reheat temperature is much larger, and that moduli dominate the energy density for some time. In this case, the inflaton may decay long before nucleosynthesis. However, unless the inflaton reheat temperature is well above $10^{11} \mathrm{GeV}$, the moduli more or less immediately come to dominate the energy density of the universe, and the estmate goes through as above. If the reheat temperature is higher (see the discussion of the next section about the gravitino problem) then if $R_{B}=n_{B} / n_{0}$, where $n_{0}$ is the entropy density just after inflation, then the final baryon to photon ratio is

$$
n_{B} / n_{\gamma}=R_{B}\left(\frac{T_{R}}{M_{I}}\right)\left(\frac{T_{m}}{M_{m}}\right) .
$$

Here $T_{m}$ denotes the reheat temperature after the moduli decay, and $M_{m}$ denotes the moduli mass. So in this situation, it will be necessary that the potential in the flat direction be extremely flat, in order for coherent baryon number production to be viable.

There are other possible mechanisms for generating the baryon asymmetry which have been discussed in the literature, such as $B$-violating gravitino decays 28 In cases where there is a stable LSP, one also needs to examine LSP production 22]. These mechanisms could also be operative here. Many of the issues are reviewed in ref. [25].

\subsection{The Gravitino Problem}

All SUSY models have a potential problem with gravitino production in the early universe. Like moduli, gravitinos can dominate the energy density of the universe and ruin the predictions of nucleosynthesis. In inflationary cosmologies, gravitinos are produced in the reheating of the universe through the decay of a coherent scalar field. In conventional

inflationary scenarios, reheat temperatures greater than $10^{9} \mathrm{GeV}$ or so lead to excessive production of gravitini 29].

The reheat of the universe through modular decay which we have discussed above, is safely below this bound. However, we must also worry about the possibility of episodes of reheating that occurred prior to the period of cosmic history when axions began to 
oscillate. In particular, if we want to embed our scenario in a model of inflation, we must enquire about the reheating due to the decay of the inflaton.

One possibility is that the modulus we have already discussed is itself the inflaton. An apparent problem with this idea is that the modular energy density appears to be much smaller that conventionally required for the inflationary explanation of the magnitude of fluctuations in the cosmic microwave background. There exist models of inflation [30] in which inflation at a scale of $10^{11} \mathrm{GeV}$ can lead to microwave fluctuations of the right size. The most attractive of these models [31], actually seems to arise quite naturally in string theory at intersection points of moduli spaces. Another way to reconcile the light modulus with inflationary expectations is to assume that the modulus is a dilaton like field, on which the potential depends in an exponential fashion (in a parametrization in which the Kahler potential of the modulus is only slowly varying) in some extreme region of moduli space. Then one can imagine that inflation takes place in a region where the potential is slowly varying, but the minimum is in the extreme region. This explains the discrepancy in inflation and SUSY breaking scales [32]. One must confront the Brustein-Steinhardt problem 33 in such a scenario, but as explained in the appendix of [32 this may not be too serious.

Suppose now that the inflaton is not the modulus responsible for the Hot Big Bang. It will have a large energy density, and Planck scale couplings, giving it a reheat temperature $T_{I}$ much higher than the scale of the electroweak phase transition. Reheating will produce a gravitino number density $n_{G}$ which is initially of order $n_{g} / s \approx 10^{-4} T_{I} / m_{p}$. After inflaton reheating, the universe remains radiation dominated for a while. The gravitino energy density falls like the cube of the scale factor, but grows linearly in the inverse temperature relative to the radiation density. The modular energy density remains constant until the Hubble parameter is equal to the mass of the modulus. If we choose a decay constant $f \sim$ $m_{p}$, this occurs at a time when the total energy density of the universe, and the modular density, are approximately equal. The corresponding temperature, $T_{m}$, is roughly $T_{m} \approx$ $1 / 5 \sqrt{m m_{p}} \cdot n_{g} / s$ remains constant until the moduli finally decay. With the assumption that the reheat temperature is of order a few $\mathrm{MeV}$, the gravitinos are diluted by a factor of order $10^{14}$. The gravitino to radiation density ratio grows by another factor of 100 before 
the gravitini decay. Previous studies have shown that this ratio must be less than about

$10^{-7}$ in order for gravitino decay to preserve the products of nucleosynthesis. Thus even for $T_{I}$ of order $m_{p}$, the gravitino density is not a problem. In other words no matter what our assumptions about the nature of inflation, the scenario outlined in this section solves the gravitino problem.

\section{Small Violation of CP}

In string theory, $\mathrm{CP}$ is an exact (gauge) symmetry, so $\mathrm{CP}$ violation is inevitably spontaneous. There are numerous CP odd fields which are candidates for breaking CP. Our fundamental assumption in the present section, will be that the breaking of CP is small. In order to be precise we will work within the context of a specific model for the origin of CP violation, but we believe that our results can be generalized to other models in a straightforward way. The model that we will use was proposed by Nir and Ratazzi [14]. All CP violation can be traced back to the vacuum expectation value (VEV) of a single field $S_{3}$, with magnitude $<S_{3}>\sim \lambda^{5} M_{p} \sim 3 \times 10^{-4} M_{p} \equiv \delta M_{p}$, where $\lambda$ is the Cabibbo angle. $S_{3}$ is a singlet under continuous gauge groups, and has only nonrenormalizable couplings to fields with masses below $M_{p}$. The pattern of these couplings is determined by an abelian horizontal symmetry. In [14] it is shown that such a model can account for all $\mathrm{CP}$ violating phenomena observed (and not observed) in nature. In particular, it leads to a CKM phase of order one, and constrains supersymmetric contributions to $\mathrm{CP}$ violating phenomena to be smaller than experimental upper limits. It does not by itself solve the strong CP problem.

Now let us turn our attention to the very early universe. We make the standard inflationary assumption that at early times the energy density of the patch which becomes our universe is dominated by nearly homogeneous classical scalar fields. We will adopt string theory language and call the space of scalars the moduli space. Among these fields are the inflatons, which coordinatize a submanifold of the moduli space to which the system's trajectory is rapidly attracted, and on which it stays for many e-foldings of the universe. The energy density on this submanifold is approximately constant, and 
drives a quasi exponential expansion. We will assume that this inflaton submanifold is approximately invariant under $\mathrm{CP}$, with the violation of $\mathrm{CP}$ being no larger than that in the vacuum. Clearly this assumption is not necessary to an understanding of the small size of $\mathrm{CP}$ violation in current experiments. Its plausibility can only be judged within the context of a theory of the potential on the space of fields, a theory which does not yet exist.

In general, during an inflationary era, we may expect the inflationary energy density to depend on all of the scalar fields in the theory. This is particularly true in the context of supergravity [34. However, this argument must be reexamined in the case of axions. The very existence of the axion depends on an approximate global symmetry of the theory, or at least of the portion of moduli space in which the vacuum state resides. The latter point of view is the one indicated by string theory. Axion PQ symmetries are approximate symmetries which arise in string theory only in special regions of moduli space where effective gauge couplings are small or internal dimensions large. It is entirely plausible that these symmetries are much more strongly broken on the inflaton submanifold. For example, the couplings of standard model gauge fields might be very large on the inflationary submanifold. In the context of the models of [7] another way to break the axion symmetries in the early universe is to shrink the eleventh dimension down to the size of the other six compact dimensions. Note that the assumption of PQ symmetry breaking during inflation contrasts with our assumption of approximate CP symmetry. Needless to say, determining what actually happens requires a much better understanding of moduli dynamics than we currently possess.

How large might the axion potentials be? Consider, first, axions whose potential vanishes as $R \rightarrow \infty$. We can easily imagine that $R=\mathcal{O}(1)$ during inflation. In this case, the axion mass would be expected to be of order the Hubble constant. For example, one might expect terms in the superpotential of the form

$$
W=e^{-R} I
$$

where $I$ is the inflaton field (assumed to have a non-vanishing $F$-component). This leads 
to

$$
m_{a}^{2} \approx H e^{-R} / f_{a}^{2}
$$

For the field which at weak coupling is termed the "model-independent axion," and usually denoted $S$, one expects a similar result. This corresponds to the possibility that the QCD coupling $\left(\alpha_{s}\right)$ is of order one during inflation.

We note that there is another puzzle of supersymmetric inflationary cosmology which may be resolved by the assumption that couplings and scales were all of order one (in fundamental units) during inflation. Typical inflation models require the inflationary energy density to be much larger than the square of the SUSY breaking order parameter in the vacuum. If the low scale of SUSY breaking is explained by a small coupling, then this discrepancy is removed [32]. We have already invoked this mechanism in the previous section.

Given these assumptions, the evolution of the axion during inflation can be described simply. Let $a=0$ be the CP invariant value of the axion field. During inflation, the axion feels an effective potential which gives it an effective mass of order the Hubble constant. It is rapidly driven to a minimum of this potential, which lies at a distance $\frac{a}{f_{a}} \sim \delta$ from the origin. By the approximate CP symmetry, this is the same order of magnitude as the distance to the true minimum of the vacuum axion potential.

The postinflationary evolution of the universe depends on whether or not there are light moduli of the sort we described in the previous section. The strongest upper bound comes from assuming that there are no such moduli. As usual we assume that the reheat temperature of the inflaton is well above the QCD scale and the postinflationary universe contains only axions and radiation. This is the conventional scenario for axions, with the exception of the fact that the initial distance of the axion field from its minimum is one ten thousandth of that which is usually assumed. For the axion energy density one has [4] [5] [6]:

$$
\Omega_{a} h^{2} \approx 0.7 \theta_{o}^{2} \times\left(f / 10^{12} \mathrm{GeV}\right)^{1.18} .
$$

(There are uncertainties in these formulas of perhaps an order of magnitude.) If $\theta_{o}$, the initial value of $\theta$, is of order $10^{-3}-10^{-4}$, then we can tolerate decay constants as large as 
$m_{p}$. This is the order of magnitude of expected for $\theta_{o}$ in models of small CP violation, in which $\theta_{o} \sim \delta$.

If some portion of early cosmological history is dominated by coherent scalars with masses coming from susy breaking then we can repeat the analysis of the previous section, but with initial axion energy density smaller by a factor of $\delta^{2} \sim 10^{-7}$. Eqn. (2.3) now becomes

$$
\delta^{2} \frac{f_{a}^{2}}{m_{p}^{2}} \frac{T_{R}}{10 \mathrm{eV}} \leq 1
$$

For a given modular reheat temperature the upper bound on the axion decay constant is larger by a factor $\delta^{-1}$. For $\delta \sim 10^{-4}$, this again gives a limit of order $m_{p}$. In each case, one can choose parameters so that axions constitute the dark matter in the universe.

\section{Eleven Dimensional Physics}

Our original motivation for returning to the cosmological conundra of axions was the observation that the fit of strongly coupled heterotic string theory to available data [35] leads to a general prediction of QCD axions with large decay constants 36. We would like to devote this section to a brief discussion of the cosmology of these strongly coupled string vacua.

Four dimensional vacua of the strongly coupled $E_{8} \mathrm{X} E_{8}$ heterotic string with $N=1$ SUSY, are described by M-theory (for practical purposes eleven dimensional supergravity) compactified on a seven manifold with boundary which is a Calabi-Yau fibration over an interval. The fit [35] to the unified fine structure constant, Newton's constant, and the

unification scale, in terms of the fundamental eleven dimensional Planck scale $l_{p}$, and the geometry of the manifold is as follows. On the boundary of the manifold with standard model gauge group, the Calabi-Yau size $R$ (volume $=R^{6}$ ), which determines the unification scale, is twice $l_{p}$, and the length of the interval, $R_{11}$, is about $70 l_{p}$. The Calabi-Yau volume decreases monotonically along the interval, and as a consequence the bare coupling of the hidden $E_{8}$ is much larger than that of the standard model. In this regime, the heterotic string is more properly thought of as a membrane stretched between the two boundaries. Its tension is $\sim 70 l_{p}^{-2}$. 
Axions are modes of the three form gauge field of $M$ theory which have the form $a(x) b_{M N}\left(x^{11}\right) d x^{M} d x^{N} d x^{11}$, where $a$ depends only on the noncompact coordinates, and $b_{M N}$ is one of the harmonic $(1,1)$ forms on the Calabi-Yau manifold at $x^{11}$. These are almost pure gauge modes when $a$ is a constant. They can be written as $d C$, where $C$ is a two form which vanishes on the $E_{8}$ boundary. Thus, constant shifts of $a$ are Peccei-Quinn symmetries broken only by effects on the standard model boundary. As argued in [36], the strongest such effect is QCD.

In the limit $R_{11} \gg l_{p}$, which seems like a reasonably good approximation to the real world, the kinetic terms of the axions are given by their values in the Kaluza-Klein approximation to $\mathrm{M}$ theory dynamics. As noted in [36], the gauge coupling functions and Kahler potentials of the bulk moduli are the same in the weak and strong coupling limits, so the result is identical to that of Kim and Choi. Thus, the axion of strongly coupled heterotic string theory fits fairly well into the framework of our first scenario. We note [36] that the true QCD axion in the strongly coupled region may in fact originate as a gauge bundle modulus on the standard model boundary. In this case, the order of magnitude estimate of the axion decay constant is $l_{11}^{-1} \sim 2 \times 10^{16} \mathrm{GeV}$. We have not yet been able to understand the precise numerical factors in this formula. If the two decay constants have the same order of magnitude the QCD axion will be a linear combination of the mode coming from the three form, and the boundary modulus.

The other part of our scenario is a modulus with a decay constant of order $m_{p}$ and potential energy of order the intermediate scale. Paradoxically, although $m_{p}$ is the natural order of magnitude estimate of the decay constant of a bulk modulus we can be less than sure that such a modulus exists. The axionlike moduli seem to have much smaller decay constants. The Kahler potentials of other moduli are not easily calculable for the values of the moduli which seem to fit the data. Our scenario looks very natural in the eleven dimensional context, but we cannot calculate enough parameters to be sure that it is realized.

Another general issue which we must face is the question of whether a satisfactory model of inflation can be built in this region of moduli space. The danger is that a simpleminded estimate of density fluctuations indicates that one needs a vacuum energy of 
order $M^{4}$ with $M \sim 10^{16} \mathrm{GeV}$ in order to account for the COBE data. This is uncomfortably close to the eleven dimensional Planck scale, above which we have no description of the correct physics in the $\mathrm{M}$ theory regime. In fact, satisfactory models of inflation with vacuum energy scale lower than $M$ by an order of magnitude abound in the literature. Theoretically, the order of magnitude estimate of the maximum potential energy of bulk moduli in the $\mathrm{M}$ theory regime is (assuming modular decay constants of order $m_{p}$ ) $l_{11}^{-6} m_{p}^{-2}$, $10^{-4}$ times smaller than the eleven dimensional Planck density which marks the border of our ignorance. This gives an effective $M$ of order $2 \times 10^{15} \mathrm{GeV}$. It is conceivable then that a semiclassical model of inflation which is both theoretically and phenomenologically viable, can be built in this regime.

The results of [36] also tell us something about the question of whether the moduli whose decay gives rise to the Hot Big Bang are inflaton fields. In the strongly coupled heterotic string it proved difficult to generate a nonperturbative energy scale higher than the hidden sector scale which gives rise to SUSY breaking. One had to assume that the the vacuum value of the modulus $\mathcal{S}$ which controls the hidden sector coupling, was at small values of the coupling. A natural way [32] to explain the large vacuum energy during inflation is to assume that $\mathcal{S}$ is one of the inflatons and that inflation occurs at strong coupling where the estimate of the energy density given in the previous paragraph is valid. On the other hand, the mass and apparent couplings of $\mathcal{S}$ make it a candidate to be the progenitor of the Hot Big Bang. Thus, the eleven dimensional scenario is likely to identify the two fields, and consequently has a very low reheat temperature.

This has been a mere sketch of the cosmology of strongly coupled heterotic string theory. We hope to return to the subject when more is understood about the effective lagrangian of the important scalar fields in this region of moduli space.

\section{Conclusions}

We have exhibited two cosmological scenarios which significantly modify the bound on the axion decay constant. In combination with the results of [11], which expand the range of expected values of $f_{a}$ in superstring theory, it is now possible to claim that 
superstring axions may be compatible with all experimental and cosmological data. Our first scenario also points up the fact that the cosmological moduli problem may be much less significant than we had imagined. Moduli compatible with weak scale baryogenesis are at the limits of plausibility but nucleosynthesis is certainly not a problem. Alternate schemes for baryogenesis are available and lead to an attractive and consistent picture of the cosmology of the very early universe. It is likely that axions with decay constant $10^{16}$ $\mathrm{GeV}$ will be the dark matter in such a scenario.

Our second solution of the cosmological axion problem has less to say about superstrings and moduli, but may have more immediate implications for low energy physics.

Our analysis of the first scenario relied on the hidden sector mechanism for SUSY breaking. It is worth saying a few words about axion properties in models where SUSY is dynamically broken in sectors coupled to the standard model through gauge interactions. In such a model, all moduli which get their mass from SUSY breaking are extremely light and have lifetimes longer than the current age of the universe. There are no apparent candidates for fields whose decay would initiate the Hot Big Bang at temperatures of order the weak scale or below. Thus, the first scenario is completely untenable in such a model. Worse, all of the moduli now tend to dominate the energy density of the universe from an energy density $\frac{f^{2}}{m_{p}^{2}} F^{2}$ (here $f$ is a typical modular decay constant, and $F$ is the SUSY breaking order parameter), until long after the present era. One is forced to imagine that the true vacuum sits at a point invariant under symmetries that transform all of the light moduli. In such a case, one might invoke a version of our second scenario to force all of the moduli to their true minima during inflation. It is however much less plausible that the inflaton is invariant under the large group of symmetries required to fix all of the light moduli. If it is not, then the scenario does not work.

Finally we note that scenarios in which the very early universe was dominated by coherent scalar fields eliminate many hypothetical cosmological phase transitions. In particular, domain walls due to spontaneously broken discrete symmetries might be a problem only if they are produced in the brief period between inflaton reheat and modular dominance (in scenarios in which the inflaton is not the modulus which produces the hot big bang), or after modular reheat. 
Acknowledgments: We thank K. Choi for discussions and for calling our attention to ref. [11]. We also thank S. Thomas for important critical comments. The work of T. Banks was supported in part by the Department of Energy under grant \#DE-FG0296ER40559. The work of M. Dine is supported in part by the U.S. Department of Energy. 


\section{References}

[1] E. Witten, Phys. Lett. 149B (1984) 351.

[2] E. Witten, Nucl. Phys. B268 (1986) 79.

[3] T. Banks and M. Dine, "Coping with Strongly Coupled String Theory," Phys. Rev. D50 (1994) 7454, hep-th/9406132.

[4] J. Preskill, F. Wilczek and M. Wise, Phys. Lett. 120B (1983) 127.

[5] L. Abbott and P. Sikivie, Phys. Lett. 120B (1983) 133.

[6] M. Dine and W. Fischler, Phys. Lett. 120B(1983) 137.

[7] T. Banks and M. Dine, "Couplings and Scales in Strongly Coupled Heterotic String Theory," RU-96-27, hep-th/9605136.

[8] P.J. Steinhardt and M.S. Turner,Phys. Lett. 129B (1983) 51.

[9] M. Kawasaki, T. Moroi and T. Yanagida, "Can Decaying Particles Raise the Upperbound on the Peccei-Quinn Scale?", hep-ph/9510461.

[10] A.D. Linde, Phys.Lett. 201B (1988) 437.

[11] K. Choi and J.E. Kim, Phys. Lett. 154B (1985) 393; K. Choi and J.E. Kim, Phys. Lett. 165B (1985) 71.

[12] I Affleck and M. Dine, Nucl. Phys. B249 (1985) 361.

[13] M. Dine, L. Randall and S. Thomas, "Baryogenesis from Flat Directions of the Supersymmetric Standard Model," Nucl. Phys. B458 (1996) 291, hep-ph/9507453.

[14] Y. Nir and R. Rattazzi, "Solving the Supersymmetric CP Problem with Abelian Horizontal Symmetries," RU-96-11, hepph/9603233.

[15] K.-W. Choi, D.V. Kaplan and A.E. Nelson, Nucl. Phys. B391 (1993) 515; M. Dine, R.G. Leigh and D. MacIntire, Phys. Rev. Lett. 69 (1992) 2030.

[16] G. Dvali, "Removing the Cosmological Bound on the Axion Scale," IFUP-TH-21-95, hep-ph/9505253.

[17] K. Choi, H.B. Kim and J.E. Kim, "Axion Cosmology with a Stronger QCD in the Early Universe," hep-ph/9696372.

[18] M. Reno and D. Seckel, Phys. Rev. D37 (1988) 3441; G. Lazarides, R. Schaefer, D. Seckel and Q. Shafi, Nucl. Phys. B346 (1990) 193.

[19] P. Horava and E. Witten, "Heterotic and Type I String Dynamics From Eleven Dimensions", Nucl. Phys. B460, (1996)506, hep-th/9510209; "Eleven-Dimensional Supergravity on a Manifold with Boundary," hep-th/9603142.

[20] T. Banks and M. Dine, "Coping with Strongly Coupled String Theory," Phys. Rev. D50 (1994) 7454, hep-th/9406132

[21] E. Witten, Phys. Lett. 155B (1985) 151.

[22] L. Randall and S. Thomas, "Solving the Cosmological Moduli Problem with Weak Scale Inflation," Nucl. Phys. B449 (1995) 229, hep-ph/9407248. 
[23] T. Banks, D.B. Kaplan and A.E. Nelson, "Cosmological Implications of Dynamical Supersymmetry Breaking," Phys. Rev. D49 (1994) 779.B. de Carlos, J.A. Casas, F. Quevedo and E. Roulet, Phys. Lett. B318 (1993) 447.

[24] G.D. Coughlan, G.G. Ross, R. Holman, P. Ramond, M. Ruiz-Altaba and J.W.F. Valle, Phys. Lett. 160B (1985) 249; G.G. Ross, R. Holman, P. Ramond, Phys. Lett. 137B , 343, (1984).

[25] S. Thomas, "Baryons and Dark Matter from the Late Decay of a Supersymmetric Condensate," Phys. Lett. B356 (1995) 256, hep-ph/9506274.

[26] D.V.Nanopoulos, S.Weinberg, Phys. Rev. D20, 2484, (1979).

[27] C.S. Aulakh and R.N. Mohapatra, Phys. Lett. 119B (1982) 316; L.J. Hall and M. Suzuki, Nucl. Phys. B231 (1984) 419; F. Zwirner, Phys. Lett. 132B (1983) 103; S. Dawson, Nucl. Phys. B261 (1985) 297; R. Barbieri and A. Masiero, Nucl. Phys. B267 (1986) 679; S. Dimopoulos and L.J. Hall, Phys. Lett. B207 (1987) 210; L. Hall, Mod. Phys. Lett. A5 (1990) 467; K.S. Babu and R.N. Mohapatra, Phys. Rev. D42 (1990) 3778.

[28] J. Cline and S. Raby, Phys. Rev. D43 (1991) 1781.

[29] J. Ellis, J.E. Kim and D.V. Nanopoulos, Phys. Lett. 145B (1984) 181.

[30] A.D.Linde, Phys. Lett.259B, 38 (1991); A.R.Liddle, D.H. Lyth, Phys. Rep. 231, 1 (1993); A.D.Linde, Phys. Rev. D49, 748, (1994); E.J.Copeland, A.R.Liddle, D.H.Lyth, E.D.Stewart, D.Wands, Phys. Rev. D49, 6410, (1994); E.D.Stewart, Phys. Lett. 345B, 414, (1995).

[31] L. Randall, M. Soljacic, and A.H. Guth, "Supernatural Inflation," MIT-CTP-2499, hep-ph/9601296.

[32] T. Banks, M. Berkooz and P.J. Steinhardt, Phys. Rev. D52 (19995) 705, hepth/9501053.

[33] R. Brustein and P.J. Steinhardt, Phys. Lett. B302 (1993) 196.

[34] G. Dvali, Phys. Lett. 355B (1995) 78; M. Dine, L. Randall, S. Thomas, "Supersymmetry Breaking in the Early Universe," Phys. Rev. Lett. B75 (1995) 398, hepph/9503303. Earlier references include M. Dine, W. Fischler and D. Nemeschansky, Phys. Lett. 136B (1984) 179 and O. Bertolami and G.G. Ross, Phys. Lett. 183B (1987) 163.

[35] E. Witten, "Strong Coupling Expansion of Calabi-Yau Compactification, hep-th/9602070.

[36] T.Banks, M.Dine, "Couplings and Scales in Strongly Coupled Heterotic String Theory", hep-th/9605136. 